\title{
The Calabi's metric for the space of Kähler metrics
}

\author{
Simone CALAMAI
}

\begin{abstract}
Given any closed Kähler manifold we define, following an idea by Eugenio Calabi [8], a Riemannian metric on the space of Kähler metrics regarded as a infinite dimensional manifold. We prove several geometrical features of the resulting space, some of which we think were already known to Calabi. In particular, the space has positive constant sectional curvature and admits explicit unique smooth solutions for the Cauchy and the Dirichlet problems for the geodesic equation.
\end{abstract}

\section{Introduction and main results}

The space of Kähler metrics is the natural environment to study the problem about the existence of Extremal Kähler metrics, which goes back to E. Calabi [5] and is a central question in Kähler geometry. The space of Kähler metrics is also the setting for the Kähler-Ricci flow and the Calabi flow. Suppose $(M, \omega)$ is a closed Kähler manifold with Kähler form $\omega$. In this introduction and in the remainder we refer to the space of Kähler metrics with the following notation

$$
\mathcal{C}:=\left\{u \in C^{\infty}(M, \mathbb{R}) \mid \int_{M} e^{u} \frac{\omega^{n}}{n !}=\int_{M} \frac{\omega^{n}}{n !}\right\}
$$

This work is the proposal of a geometric structure for the space of Kähler metrics viewed as an infinite dimensional manifold. Another geometry for the same space turned out to be decisive to affirmatively answer the question of uniqueness of Extremal metrics. For that, great contributions were given by Xiu Xiong Chen [9] and S. Donaldson [15]. In that case, the geometry arises from a metric which we call the Mabuchi-Semmes-Donaldson's metric, since those three authors defined it and contributed to its study. Our geometry arises from an intuition by E. Calabi, as we learned by Xiu Xiong Chen [8]. The expression of the Calabi's metric is

$$
<v, w>_{u}=\int_{M} v w e^{u} \frac{\omega^{n}}{n !}, \quad v, w \in T_{u} \mathcal{C} .
$$


The purpose of this paper is to compare those two geometries; hereafter we summarize the main results. For the first one, compare Theorems 3.4, 4.4 of the present paper.

Theorem 1.1 Let $(M, \omega)$ a closed Kähler manifold. The Calabi's metric admits the Levi Civita covariant derivative; its sectional curvature is positive, constant and equal to $s=\frac{1}{4 \mathrm{Vol}}$, where $\mathrm{Vol}$ is the volume of the manifold $M$. As a consequence, the space of Kähler metrics is a locally symmetric space.

In his seminal paper, T. Mabuchi [17] got similar results for his metric; i.e. he explicitly computed the Levi Civita covariant derivative which he proved to entail a structure of locally symmetric space. But he proved also that his metric has non positive sectional curvature. On this aspect, that metric seems to be opposite to the Calabi's one, and of course the two models are not isometric. The Calabi's metric case already showed the pleasant feature to have constant curvature; more pleasant features comes with the analysis of the geodesic equation (cfr. Theorem $5.2)$.

Theorem 1.2 The geodesic equation is equivalent to an ordinary differential equation, namely to the equation

$$
\left(e^{\frac{u}{2}}\right)_{t t}+e^{\frac{u}{2}}=0
$$

On this side, our case is the best one can hope for and again very different from the other one. There, unaware f the Mabuchi's paper, S. Semmes [20] rediscovered the same metric via a totally different line of reasoning. Indeed, he was studying the Homogeneous Complex Monge-Ampère equation on a complex domain $D \subset \mathbb{C}^{n}$ and proved that in some special cases it is a geodesic equation for the space of plurisubharmonic functions on $D$. Moreover, he was able to construct a metric on the space of plurisubharmonic functions on $D$ such that its corresponding geodesic equation is precisely the Homogeneous Complex Monge-Ampère equation. In [15], S. Donaldson made precise that the geodesic equation for the MabuchiSemmes-Donaldson's metric is equivalent to a Homogeneous Complex MongeAmpère equation. What really interests us is not the complexity of that equation, but rather the consequences on the geometry; these appear in the next result (cfr. Theorems 5.2,5.4 and 7.10).

Theorem 1.3 The Cauchy problem for the geodesic equation has unique real analytic solutions for any initial data; the explicit expression of a solution is

$$
u(t)=u_{0}+2 \log \left(\cos (t)+\frac{v_{0}}{2} \sin (t)\right) .
$$

Moreover, this allows to define the exponential map, which is injective and surjective. Finally, the space of Kähler metrics does not have conjugate points. 
In [15], S. Donaldson showed examples of nonexistence of solutions for the Cauchy problem in the Mabuchi-Semmes-Donaldson's setting. Thus, the notions of exponential map and conjugate points couldn't be carried over. On this aspect, we may say that the Calabi's geometry is richer than that one. This appears also in the next result (cfr. Theorems 6.1, 6.4, 6.6).

Theorem 1.4 The Dirichlet problem for the geodesic equation has unique real analytic solutions for any boundary data $u_{0}, u_{1} \in \mathcal{C}$; the explicit expression of a solution is

$$
e^{\frac{u(t)}{2}}=e^{\frac{u_{0}}{2}}\left(\cos (t)+e^{\frac{u_{1}-u_{0}}{2}} \frac{\sin (t)}{\sin \left(t_{0}\right)}-\cos \left(t_{0}\right) \frac{\sin (t)}{\sin \left(t_{0}\right)}\right)
$$

The space of Kähler metrics is a metric space with distance function real analytic. Geodesics are minima of the length functional. Moreover, the diameter of the space is $\frac{\pi}{2} R$, where the sectional curvature is considered as $s=\frac{1}{R^{2}}$.

In [9], Xiu Xiong Chen solved the Dirichlet problem for geodesics in the MabuchiSemmes-Donaldson's setting. Those geodesic segments, called Chen's geodesics, are of class $C^{1,1}$ and they stay in the closure of the space of Kähler metrics. The improvement of the latter two facts is still an open problem. Moreover, Chen showed that the space is a metric space with distance function of class $C^{1}$, and that geodesics minimize the length functional. We mention that these results led to prove the uniqueness of constant scalar curvature metrics; this was done by Xiu Xiong Chen [9] and by Xiu Xiong Chen and Gang Tian [14]. Other important results are: E. Calabi and Xiu Xiong Chen [7] proved that the space of Kähler potentials endowed with the Mabuchi-Semmes-Donaldson's metric is non positively curved in the sense of Alexandrov and Xiu Xiong Chen [10] proved the inequality involving the geodesic distance induced by the Mabuchi-SemmesDonaldson's metric $E\left(\phi_{1}\right)-E\left(\phi_{0}\right) \leq \sqrt{\operatorname{Cal}\left(\phi_{1}\right)} d\left(\phi_{0}, \phi_{1}\right)$. Recently, Xiu Xiong Chen and Song Sun [13] reproved the latter two results using a quantization of the space of Kähler potentials. About the question on the diameter, the two situations seems to be opposite; in fact Donaldson showed how the study of geodesic rays in the space of Kähler potentials endowed with the Mabuchi-Semmes-Donaldson's metric that would lead to results about the existence of Extremal metrics (on this aspect, see [1] and [11]). In the Calabi's case, the fact that the sectional curvature is constant and positive, the fact that there are no conjugate points and the explicit value of the diameter led to the result which summarize the present study of the geometry of the Calabi's metric. (cfr. Theorems 7.12, 6.9).

Theorem 1.5 There is an isometric immersion of the space of Kähler metrics into the space of real valued smooth functions on $M$ endowed with the Euclidean metric, and the image of the space $\mathcal{C}$ under this immersion is a portion of an infinite dimensional sphere. Moreover, the distance to the boundary of any point of the space is zero. 
Recently the interest for Riemannian metrics in infinite dimensional spaces has been renewed by S. Donaldson [16] who generalized the considerations made in the Kähler case to the Riemannian case, i.e. when is given a closed Riemannian manifold $(M, g)$ neither necessarily Kähler nor complex. In this more general case the Riemann metric is defined no more in a fixed Kähler class but in the space of volume forms which are conformal to the given one $d \mu$ and which have the same area;

$$
V:=\left\{f \in C^{\infty}(M,(0,+\infty)) \mid \int_{M} f d \mu=\int_{M} d \mu\right\} .
$$

The space $V$ can be parameterized as

$$
\mathcal{S}:=\left\{\phi \in C^{\infty}(M, \mathbb{R}) \mid 1+\Delta_{g} \phi>0\right\}
$$

and the metric in $\mathcal{S}$ is defined by

$$
<\psi, \chi>_{\phi}:=\int_{M} \psi \chi\left(1+\Delta_{g} \phi\right) d \mu .
$$

When $(M, g)$ has real dimension 2 this metric coincides with the Mabuchi-SemmesDonaldson's metric. Endowed with this metric by Donaldson, $V$ has a very interesting geometry structure as well. The existence and the study of the geometry arising from Donaldson's metric on $V$ is due to Xiu Xiong Chen and Weiyong He (see [12] for more details), and their arguments generalize the techniques used by Chen in [9].

We remark here that we can generalize the Calabi's metric to the space $V$; in fact we parameterize the space $V$ as

$$
\mathcal{C}:=\left\{u \in C^{\infty}(M, \mathbb{R}) \mid \int_{M} e^{u} d \mu=\int_{M} d \mu\right\}
$$

and we define the metric

$$
<v, w>_{u}:=\int_{M} v w e^{u} d \mu .
$$

We claim that the above definition coincides with the Calabi's metric. In fact, in the Kähler case, the Calabi's volume conjecture [4] solved by S.T. Yau [22] entails that the space of metrics in the anti-canonical bundle of the given Kähler manifold is equivalent to the space of Kähler potentials up to a constant; moreover it is not hard to see that through the above equivalence the Calabi's metric translates into a $L^{2}$ metric on the space of metrics in the anti-canonical bundle of the given Kähler manifold.

An interesting reference for a further generalization of the Calabi's metric are the recent lecture notes by Bourguignon [3], which relates together spaces of probability theory, optimal transportation theory and Riemannian geometry.

Acknowledgements: I want to thank my advisor Giorgio Patrizio, Claudio 
Arezzo and Xiu Xiong Chen for his support. Thanks also to Chen's group of students, in particular to Song Sun for many helpful discussions and his interest on this problem. Part of this work was written at UW-Madison and at USTC, Hefei. I thank both those Universities for their hospitality.

\section{The Calabi's metric}

In this section we introduce the Calabi's metric. For the reader's convenience, we recall some well known definitions which lead to that notion. From now on, $(M, \omega)$ is a closed Kähler manifold of complex dimension $n$ with Kähler form $\omega$.

Definition 2.1 The space of volume conformal factors of $(M, \omega)$ is

$$
\mathcal{C}:=\left\{u \in C^{\infty}(M, \mathbb{R}) \mid \int_{M} e^{u} \frac{\omega^{n}}{n !}=\int_{M} \frac{\omega^{n}}{n !}\right\}
$$

The space of Kähler potentials $\mathcal{H}$ is $\mathcal{H}:=\left\{\phi \in C^{\infty}(M, \mathbb{R}) \mid \omega+i \partial \bar{\partial} \phi>0\right\}$. The space $\tilde{\mathcal{H}}$ of normalized potentials is given by $\tilde{\mathcal{H}}:=\{\phi \in \mathcal{H} \mid L(0, \phi)=0\}$, where $L(\rho, \eta):=\left.\frac{1}{V o l} \int_{0}^{1}\left(\int_{M} \phi \frac{\omega_{s \phi}^{n}}{n !}\right) d s\right|_{\phi=\rho} ^{\phi=\eta}$ and $\rho, \eta \in \mathcal{H}$, according to [17] and [18].

Remark 2.2 The space $\mathcal{C}$ is diffeomorphic to the space $\tilde{\mathcal{H}}$; indeed the map Cal : $\tilde{\mathcal{H}} \rightarrow \mathcal{C}, \operatorname{Cal}(\phi)=\log \left(\frac{\omega_{\phi}^{n}}{\omega^{n}}\right)$ is a diffeomorphism (see [2]) according to the Calabi's conjecture [4] solved by Yau [22].

Definition 2.3 A smooth curve in $\mathcal{C}$ is a map $u=u(t):(-\epsilon, \epsilon) \rightarrow \mathcal{C}$ such that the map

$$
\begin{array}{ccc}
u:(-\epsilon, \epsilon) \times M & \rightarrow & \mathbb{R} \\
(t, p) & \mapsto & (u(t))(p) .
\end{array}
$$

is smooth.

Definition 2.4 Fix a point $u \in \mathcal{C}$. Let $\alpha:(-\epsilon, \epsilon) \rightarrow \mathcal{C}$ a smooth curve with $\alpha(0)=u$. Then $\left.\frac{d \alpha}{d t}\right|_{t=0}$ is a tangent vector at $u$. The whole tangent vectors at $u$ form $T_{u} \mathcal{C}$, the tangent space at $u$. A similar definition holds for $\mathcal{H}$ and $\tilde{\mathcal{H}}$.

Proposition 2.5 The following characterization of the tangent space at $u$ holds

$$
T_{u} \mathcal{C}=\left\{v \in C^{\infty}(M, \mathbb{R}) \mid \int_{M} v e^{u} \frac{\omega^{n}}{n !}=0\right\}
$$

Similarly, $T_{\phi} \tilde{\mathcal{H}}=\left\{\psi \in C^{\infty}(M, \mathbb{R}) \mid \int_{M} \psi \frac{\omega_{\phi}^{n}}{n !}=0\right\}$.

Definition 2.6 Let $u=u(t):(-\epsilon, \epsilon) \rightarrow \mathcal{C}$ be a smooth curve; a real function $v \in C^{\infty}((-\epsilon, \epsilon) \times M, \mathbb{R})$ is a smooth section on $u$ when $v(t, \cdot) \in T_{u(t)} \mathcal{C}$ for any $t$. 
Definition 2.7 A metric or 'Riemannian metric' on $\mathcal{C}$ is a positive bilinear form at any tangent space of $\mathcal{C}$, which is differentiable along any smooth sections on any smooth curve on $\mathcal{C}$.

Definition 2.8 The Calabi's metric is given by, at any $u \in \mathcal{C}$,

$$
\mathrm{Ca}(v, w)=<v, w>_{u}:=\int_{M} v w e^{u} \frac{\omega^{n}}{n !}, \quad v, w \in T_{u} \mathcal{C} .
$$

Remark 2.9 The Calabi's metric is an idea by Eugenio Calabi [8] and we learned it from Xiu Xiong Chen. It satisfies the requirements of the Definition 2.7.

Comparison with the Mabuchi-Semmes-Donaldson metric 2.10 The map Cal recalled in Remark 2.2 is an isometry between $(\mathcal{C}, \mathrm{Ca})$ and the space $\tilde{\mathcal{H}}$ endowed with the metric

$$
<\psi, \chi>_{\phi}=\int_{M}\left(\Delta_{\phi} \psi\right)\left(\Delta_{\phi} \chi\right) \frac{\omega_{\phi}^{n}}{n !}, \quad \psi, \chi \in T_{\phi} \tilde{\mathcal{H}}
$$

the Mabuchi-Semmes-Donaldson metric is (isometric to)

$$
\prec \psi, \chi \succ_{\phi}:=\int_{M} \psi \chi \frac{\omega_{\phi}^{n}}{n !}, \quad \psi, \chi \in T_{\phi} \tilde{\mathcal{H}} .
$$

So the Calabi's metric is a $L^{2}$ pairing of the Laplacian instead of just a $L^{2}$ pairing.

\section{The Levi Civita covariant derivative}

In this section we compute the explicit expression of the Levi Civita covariant derivative for the Calabi's metric. This a fortiori proves its existence which in this infinite dimensional setting is not guaranteed by the standard finite dimensional argument (cfr. [15]).

Definition 3.1 The Levi Civita covariant derivative for the space $\mathcal{C}$ endowed with a 'Riemannian metric' $g$ is a map defined on every smooth curve $u=u(t)$ : $(-\epsilon, \epsilon) \rightarrow \mathcal{C}$ and a smooth real section $v$ along $u$. The Levi Civita covariant derivative of $v$ along $u$ is denoted by $D_{t} v$ and it is a smooth section along $u$. It is required to satisfy

$$
\begin{aligned}
& \text { (i) } D_{t}(v+w)=D_{t} v+D_{t} w ; \\
& \text { (ii) } D_{t}(f v)=f D_{t} v+\frac{d}{d t} f v ; \\
& \text { (iii) } \frac{d}{d t} g_{\phi}(v, w)=g_{\phi}\left(D_{t} v, w\right)+g_{u}\left(v, D_{t} w\right) ; \\
& \text { (iv) } \tau(\alpha):=D_{t} \frac{\partial \alpha}{\partial s}-D_{s} \frac{\partial \alpha}{\partial t}=0
\end{aligned}
$$

where, along the path $u$, are given $v, w$ smooth sections and $f$ is a smooth function; $\alpha$ is a smooth two parameter family in $\mathcal{C}$ and $\tau$ is the torsion. 
Remark 3.2 The requirement for the Levi Civita covariant derivative to be torsion free finds an application in those computations where there is a smooth two parameter family in the space of Kähler metrics; we are allowed to switch in this sense $D_{t} \frac{\partial \alpha}{\partial s}=D_{s} \frac{\partial \alpha}{\partial t}$, as follows from the fact that $D$ is torsion free.

Remark 3.3 We used the expression 'the Levi Civita covariant derivative'. In fact it holds both in the finite dimensional and in this infinite dimensional case that the Levi Civita covariant derivative, if it exists, is unique (cfr. [15]). For a finite dimensional Riemannian manifold the Fundamental lemma of Riemannian geometry (see [19]) guarantees the existence of the Levi Civita covariant derivative; that argument does not work in our infinite dimensional setting (cfr. [15]). This motivates the next result.

Theorem 3.4 The Levi Civita covariant derivative for the space $\mathcal{C}$ endowed with the Calabi's metric exists; moreover its explicit expression is

$$
D_{t} v=v^{\prime}+\frac{1}{2} v u^{\prime}+\frac{1}{2 V o l} \int_{M} v u^{\prime} e^{u} \frac{\omega^{n}}{n !},
$$

where $u=u(t):(-\epsilon, \epsilon) \rightarrow \mathcal{C}$ is a smooth path and $v=v(t)$ is a smooth section along $u$.

Proof. It is sufficient to prove the second part of the statement. Let $u, v, w, f$ be as in Definition 3.1. To prove that $D_{t} v$ is a smooth section along $u$ it is enough to show that $\int_{M}\left(D_{t} v\right) e^{u \frac{\omega^{n}}{n !}}=0$;

$$
\begin{aligned}
& \int_{M}\left(\frac{d}{d t} v+\frac{1}{2} \frac{d}{d t} u v+\frac{1}{2 V o l} \int_{M} v \frac{d}{d t} u \frac{\omega^{n}}{n !}\right) e^{u} \frac{\omega^{n}}{n !}= \\
= & \int_{M} \frac{d}{d t} v e^{u} \frac{\omega^{n}}{n !}+\int_{M} v \frac{d}{d t} u e^{u} \frac{\omega^{n}}{n !}=\frac{d}{d t} \int_{M} v e^{u} \frac{\omega^{n}}{n !}=0,
\end{aligned}
$$

as claimed. The following computation

$$
\begin{gathered}
D_{t}(v+w)=\frac{d}{d t}(v+w)+\frac{1}{2}(v+w) \frac{d}{d t} u+\frac{1}{2 V o l} \int_{M}(v+w) \frac{d}{d t} u \frac{\omega^{n}}{n !}= \\
=\left(\frac{d}{d t} v+\frac{1}{2} v \frac{d}{d t} u+\frac{1}{2 V o l} \int_{M} v \frac{d}{d t} u \frac{\omega^{n}}{n !}\right)+\left(\frac{d}{d t} w+\frac{1}{2} w \frac{d}{d t} u+\frac{1}{2 V o l} \int_{M} w \frac{d}{d t} u \frac{\omega^{n}}{n !}\right)=D_{t} v+D_{t} w
\end{gathered}
$$

proves (i) of Definition 3.1. About (ii),

$$
\begin{gathered}
D_{t}(f v)=\frac{d}{d t}(f v)+\frac{1}{2}(f v) \frac{d}{d t} u+\frac{1}{2 V o l} \int_{M}(f v) \frac{d}{d t} u \frac{\omega^{n}}{n !}= \\
=v \frac{d}{d t} f+\left(f \frac{d}{d t} v+f \frac{1}{2} v \frac{d}{d t} u+f \frac{1}{2 V o l} \int_{M} v \frac{d}{d t} u \frac{\omega^{n}}{n !}\right)=v \frac{d}{d t} f+f D_{t} v,
\end{gathered}
$$


as required, where is used that $f$ does not depend on spacial variables. To check (iii) with $g$ being the Calabi's metric it is enough to show

$$
\frac{d}{d t} \int_{M} v^{2} e^{u} \frac{\omega^{n}}{n !}=2 \int_{M} v\left(D_{t} v\right) e^{u} \frac{\omega^{n}}{n !} .
$$

The above equality holds since

$$
\begin{gathered}
\frac{d}{d t} \int_{M} v^{2} e^{u} \frac{\omega^{n}}{n !}=\int_{M}\left(2 v \frac{d}{d t} v+v^{2} \frac{d}{d t} u\right) e^{u} \frac{\omega^{n}}{n !}= \\
=2 \int_{M} v\left(\frac{d}{d t} v+\frac{1}{2} v \frac{d}{d t} u+\frac{1}{2 V o l} \int_{M}\right) e^{u} \frac{\omega^{n}}{n !}-\int_{M} v \frac{1}{V o l}<v, \frac{d}{d t} u>_{u} e^{u} \frac{\omega^{n}}{n !}= \\
=2 \int_{M} v\left(D_{t} v\right) e^{u} \frac{\omega^{n}}{n !}-\frac{1}{V o l}<v, \frac{d}{d t} u>_{u} \int_{M} v e^{u} \frac{\omega^{n}}{n !}=2 \int_{M} v\left(D_{t} v\right) e^{u} \frac{\omega^{n}}{n !}-0 .
\end{gathered}
$$

Finally, about $(i v)$, let $\alpha=\alpha(s, t)$ be a smooth two parameter family. The computation

$$
D_{t} \frac{\partial \alpha}{\partial s}=\frac{\partial^{2} \alpha}{\partial t \partial s}+\frac{1}{2} \frac{\partial \alpha}{\partial s} \frac{\partial \alpha}{\partial t}+\frac{1}{2 V o l}<\frac{\partial \alpha}{\partial s}, \frac{\partial \alpha}{\partial t}>_{\alpha}=D_{s} \frac{\partial \alpha}{\partial t}
$$

completes the proof.

\section{The Calabi's metric has positive constant sectional curvature}

In [8] Eugenio Calabi was aware of the fact that his metric has positive curvature. In this section we prove that the space $\mathcal{C}$ endowed with the Calabi's metric has sectional curvature equal to $(4 \mathrm{Vol})^{-1}$, where $\mathrm{Vol}=\int_{M} \frac{\omega^{n}}{n !}$ is the volume of $M$. As a consequence, the space is locally symmetric, that is the covariant derivative of the curvature tensor is identically zero, and the Calabi's metric is not isometric to the Mabuchi-Semmes-Donaldson metric. For the sake of notations, we will write $u_{s}$ for $\frac{\partial u}{\partial s}$ and also for $\left.\frac{\partial u}{\partial s}\right|_{s=0}$ when no confusion arises; moreover we will write $\langle\cdot, \cdot\rangle$ for $\langle\cdot, \cdot\rangle_{u}$, i.e. with the omission of $u$.

Definition 4.1 Let $g$ be a metric on $\mathcal{C}$ with Levi Civita covariant derivative $D$; the curvature tensor is defined on a four parameter family $u(q, r, s, t)$ by

$$
R\left(u_{q}, u_{r}, u_{s}, u_{t}\right):=g\left(R\left(u_{q}, u_{r}\right) u_{s}, u_{t}\right),
$$

where $R\left(u_{q}, u_{r}\right) u_{s}:=\left(D_{q} D_{r}-D_{r} D_{q}\right) u_{s}$. Its covariant derivative is a 5 -tensor given by

$D R\left(u_{p}, u_{q}, u_{r}, u_{s}, u_{t}\right):=D_{p} R\left(u_{q}, u_{r}, u_{s}, u_{t}\right)-R\left(D_{p} u_{q}, u_{r}, u_{s}, u_{t}\right)-\cdots-R\left(u_{q}, u_{r}, u_{s}, D_{p} u_{t}\right)$. 
The sectional curvature of the plane $P:=\mathbb{R} \frac{\partial u}{\partial s}+\mathbb{R} \frac{\partial u}{\partial t}$ is given by

$$
K(P):=-\frac{g\left(R\left(u_{s}, u_{t}\right) u_{s}, u_{t}\right)}{g\left(u_{t}, u_{t}\right) g\left(u_{s}, u_{s}\right)-g\left(u_{s}, u_{t}\right)^{2}} .
$$

Theorem 4.2 Let $u=u(q, r, s, t)$ a four parameter family in $\mathcal{C} ; u:(-\epsilon, \epsilon)^{4} \rightarrow \mathcal{C}$. The curvature tensor for the Calabi's metric is

$$
R\left(u_{q}, u_{r}, u_{s}, u_{t}\right)=\frac{1}{4 \mathrm{Vol}}\left(<u_{r}, u_{s}>_{u}<u_{q}, u_{t}>_{u}-<u_{q}, u_{s}>_{u}<u_{r}, u_{t}>_{u}\right) .
$$

Proof. It is convenient to compute first $D_{q} D_{r} u_{s}$. By definition

$$
\begin{gathered}
D_{q} D_{r} u_{s}=D_{q}\left(u_{s r}+\frac{1}{2} u_{s} u_{r}+\frac{1}{2 \mathrm{Vol}}<u_{s}, u_{r}>\right)= \\
=u_{s r q}+\frac{1}{2} u_{s r} u_{q}+\frac{1}{2 \mathrm{Vol}}<u_{s r}, u_{q}>+\frac{1}{2}\left(u_{s} u_{r}\right)_{q}+\frac{1}{4} u_{s} u_{r} u_{q}+\frac{1}{4 \mathrm{Vol}}<u_{s} u_{r}, u_{q}>+ \\
+\frac{1}{2 \mathrm{Vol}} \frac{\partial}{\partial q}<u_{s}, u_{r}>+\frac{1}{4 \mathrm{Vol}}<u_{s}, u_{r}>u_{q}+\frac{1}{4 \mathrm{Vol}^{2}}<<u_{s}, u_{r}>, u_{q}>= \\
=u_{s r q}+\frac{1}{2}\left(u_{s r} u_{q}+u_{s q} u_{r}\right)+\frac{1}{2} u_{s} u_{r q}+\frac{1}{4} u_{s} u_{r} u_{q}+\frac{1}{2 \mathrm{Vol}}<u_{s r}, u_{q}>+ \\
+\frac{1}{4 \mathrm{Vol}}<u_{s} u_{r}, u_{q}>+\frac{1}{2 \mathrm{Vol}}<u_{s q}+\frac{1}{2} u_{s} u_{q}+\frac{1}{2 \mathrm{Vol}}<u_{s}, u_{q}>, u_{r}>+ \\
+\frac{1}{2 \mathrm{Vol}}<u_{s}, u_{q r}+\frac{1}{2} u_{r} u_{q}+\frac{1}{2 \mathrm{Vol}}<u_{r}, u_{q}>+\frac{1}{4 \mathrm{Vol}}<u_{s}, u_{r}>u_{q}= \\
=u_{s r q}+\frac{1}{2}\left(u_{s r} u_{q}+u_{s q} u_{r}\right)+\frac{1}{2} u_{s} u_{r q}+\frac{1}{4} u_{s} u_{r} u_{q}+ \\
+\frac{1}{2 \mathrm{Vol}}\left(<u_{s r}, u_{q}>+<u_{s q}, u_{r}>\right)+\frac{1}{4 \mathrm{Vol}_{2}}\left(<u_{s} u_{r}, u_{q}>+<u_{s} u_{q}, u_{r}>\right)+ \\
+\frac{1}{2 \mathrm{Vol}}<u_{s}, u_{q r}>+\frac{1}{2 \mathrm{Vol}}<u_{s}, u_{q} u_{r}>+\frac{1}{4 \mathrm{Vol}}<u_{r}, u_{s}>u_{q} .
\end{gathered}
$$

The above computation leads to this explicit expression

$$
R\left(u_{q}, u_{r}\right) u_{s}=D_{q} D_{r} u_{s}-D_{r} D_{q} u_{s}=\frac{1}{4 \mathrm{Vol}}\left(<u_{r}, u_{s}>u_{q}-<u_{q}, u_{s}>u_{r}\right),
$$

form which the claim of the theorem easily follows.

This first consequence of the Theorem 4.2 is immediate.

Corollary 4.3 The curvature tensor for the Calabi's metric is locally symmetric, i.e. $D R=0$.

The following consequence of the previous theorem is so important that we label again as a theorem its statement. 
Theorem 4.4 The curvature of the space $\mathcal{C}$ endowed with the Calabi's metric is $\frac{1}{4 \mathrm{Vol}}$, i.e. it is positive, constant and it depends only on the volume of the manifold $(M, \omega) \square$.

Comparison with the Mabuchi-Semmes-Donaldson metric 4.5 Both metrics induce a structure of locally symmetric space, i.e. their curvature tensor is covariant constant (see [15]). Mabuchi in [17] proved his metric to have non positive sectional curvature; Theorem 4.4 entails that the two metrics are not isometric.

Remark 4.6 We will often employ the notation $R^{2}=\frac{1}{4 \text { Vol }}$; the normalization $R=1$ corresponds to replace $\omega$ with $\frac{1}{\sqrt[n]{4 \text { Vol }}} \omega$.

\section{The Cauchy problem for the geodesic equation}

In this section we prove that the Cauchy problem for the geodesic equation admits, for any initial datum, an explicit unique smooth solution; as remarked by S. Donaldson in [15], this does not happen for the Mabuchi-Semmes-Donaldson metric. We define the exponential map and we prove that it is a diffeomorphism on its domain. We point out that in our case the geodesic equation reduces to a ordinary differential equation, while for the Mabuchi-Semmes-Donaldson metric it is equivalent to a Monge-Ampère equation.

Definition 5.1 Let $g$ be 'Riemannian metric' for the space $\mathcal{C}$. Assume that $\nabla_{t}$ is the Levi Civita covariant derivative induced by the metric $g$. A path $\phi=\phi(t)$ is said to be a geodesic curve if it satisfies the geodesic equation

$$
\nabla_{t} \frac{\partial \phi}{\partial t}=0
$$

Theorem 5.2 Let $u_{0} \in \mathcal{C}$ and $v_{0} \in T_{u_{0}} \mathcal{C}$ be respectively the given initial position and initial velocity. Then there exists a unique geodesic curve $u=u(t)$ in $\mathcal{C}$ satisfying the Cauchy problem

$$
\left\{\begin{array}{c}
D_{t} u^{\prime}=0 \\
u(0)=u_{0} \\
u^{\prime}(0)=v_{0} .
\end{array}\right.
$$

The curve is smooth and, if $v_{0} \neq 0$, is given by

$$
u(t)=u_{0}+2 \log \left(\cos \left(\frac{\left|v_{0}\right|_{u_{0}}}{R} t\right)+\frac{v_{0}}{2} \frac{R}{\left|v_{0}\right|_{u_{0}}} \sin \left(\frac{\left|v_{0}\right|_{u_{0}}}{R} t\right)\right)
$$


it is defined for those values of $t$ in the open interval

$$
\left(-\frac{R}{\left|v_{0}\right|_{u_{0}}} \operatorname{arccot}\left(\frac{\max v_{0}}{2} \frac{R}{\left|v_{0}\right|_{u_{0}}}\right), \frac{R}{\left|v_{0}\right|_{u_{0}}} \operatorname{arccot}\left(\frac{-\min v_{0}}{2} \frac{R}{\left|v_{0}\right|_{u_{0}}}\right)\right),
$$

where the function arccot is meant to range in the interval $(0, \pi)$.

Proof. Fix $R=1$, so that the geodesic equation is $u^{\prime \prime}+\frac{1}{2}\left(u^{\prime}\right)^{2}+2<u^{\prime}, u^{\prime}>=0$. A first claim is that along a geodesic $<u^{\prime}, u^{\prime}>(t)=<u^{\prime}, u^{\prime}>(0)$; in fact $\frac{d}{d t}<u^{\prime}, u^{\prime}>=2<D u^{\prime}, u^{\prime}>=0$ by the metric compatibility. Introduce the notation $\left|v_{0}\right|_{u_{0}}:=<u^{\prime}, u^{\prime}>(0)$; the geodesic equation is thus proved to be equivalent to the second order ordinary differential equation

$$
u^{\prime \prime}+\frac{1}{2}\left(u^{\prime}\right)^{2}+2\left|v_{0}\right|_{u_{0}}^{2}=0 .
$$

This is in fact the harmonic oscillator equation of proper frequency $\left|v_{0}\right|_{u_{0}}$ in the unknown $e^{\frac{u}{2}}$; indeed $\left(e^{\frac{u}{2}}\right)^{\prime \prime}=\frac{1}{2} e^{\frac{u}{2}}\left(u^{\prime \prime}+\frac{1}{2}\left(u^{\prime}\right)^{2}\right)$, so that the geodesic equation is equivalent to

$$
\left(e^{\frac{u}{2}}\right)^{\prime \prime}+\left|v_{0}\right|_{u_{0}}^{2} e^{\frac{u}{2}}=0,
$$

having multiplied it by the positive factor $\frac{1}{2} e^{\frac{u}{2}}$. About the corresponding Cauchy problem, in the case that $v_{0}=0$ readily the unique solution is $u(t)=u_{0}$ for any $t$; if $\left|v_{0}\right|_{u_{0}}>0$, the unique solution is the smooth curve

$$
e^{\frac{u}{2}}=e^{\frac{u_{0}}{2}}\left(\cos \left(\left|v_{0}\right|_{u_{0}} t\right)+\frac{v_{0}}{2\left|v_{0}\right|_{u_{0}}} \sin \left(\left|v_{0}\right|_{u_{0}} t\right)\right),
$$

which is equivalent to that one in the statement. Notice that the solution makes sense as long as $\left(\cos \left(\left|v_{0}\right|_{u_{0}} t\right)+\frac{v_{0}}{2\left|v_{0}\right|_{0}} \sin \left(\left|v_{0}\right|_{u_{0}} t\right)\right)>0$, which gives the interval of definition of the geodesic that is written in the statement. The case when $R \neq 1$ follows exactly from the same argument.

Motivated by the last result we try a definition of the exponential map.

Definition 5.3 Let $\gamma\left(t, u_{0}, v_{0}\right) \in \mathcal{C}$ be the point reached after a time $t$ by the geodesic with initial data $\left(u_{0}, v_{0}\right)$. The exponential map at $u_{0}$ assigns to $v_{0} \in T_{u_{0}} \mathcal{C}$ the quantity $\exp _{u_{0}}\left(v_{0}\right)=\gamma\left(1, u_{0}, v_{0}\right)$ whenever it makes sense, i.e. in

$$
D_{u_{0}}:=\left\{\left.v \in T_{u_{0}} \mathcal{C}|| v\right|_{u_{0}}<\operatorname{arccot}\left(\frac{-\min v}{2|v|_{u_{0}}}\right)\right\}
$$

Theorem 5.4 For any $u_{0} \in \mathcal{C}$, the exponential map $\exp _{u_{0}}: D_{u_{0}} \rightarrow \mathcal{C}$ is injective and surjective. 
Proof. About the injectivity, first notice that $\exp _{u_{0}}(v)=u_{0}$ if and only if $v=0$. Then suppose $\exp _{u_{0}}(v)=\exp _{u_{0}}(w)$ with $v, w \neq 0$, i.e.

$$
\cos \left(|v|_{u_{0}}\right)+\frac{v}{2|v|_{u_{0}}} \sin \left(|v|_{u_{0}}\right)=\cos \left(|w|_{u_{0}}\right)+\frac{w}{2|w|_{u_{0}}} \sin \left(|w|_{u_{0}}\right) .
$$

An integration gives $\cos \left(|v|_{u_{0}}\right)=\cos \left(|w|_{u_{0}}\right)$; since any nonzero $v \in D_{u_{0}}$ satisfies $0<|v|_{u_{0}} \leq \frac{\pi}{2}$ that entails $|v|_{u_{0}}=|w|_{u_{0}}$. The hypothesis now writes as $(v-$ $w) \frac{\sin \left(|v|_{u_{0}}\right)}{2|v| u_{0}}=0$, that gives $v=w$ as claimed. To see that $\exp _{u_{0}}$ is surjective, let $w \in \mathcal{C}, w \neq u_{0}$ be a generic element. It is required to prove that there is a solution $0 \neq v \in D_{u_{0}}$ for the equation $e^{\frac{u_{0}}{2}}\left(\cos \left(|v|_{u_{0}}\right)+\frac{v}{2|v|_{u_{0}}} \sin \left(|v|_{u_{0}}\right)\right)=e^{\frac{w}{2}}$. Multiply by $e^{\frac{u_{0}}{2}}$ and integrate to get $\cos \left(|v|_{u_{0}}\right)=4 \int_{M} e^{\frac{u_{0}+w}{2}} \frac{\omega^{n}}{n !} \in(0,1)$. Insert the last result in the first equation to get this expression of $v$ in terms of $u_{0}$ and $w$

$$
v=\left(e^{\frac{w-u_{0}}{2}}-4 \int_{M} e^{\frac{u_{0}+w}{2}} \frac{\omega^{n}}{n !}\right) \frac{2 \arccos \left(4 \int_{M} e^{\frac{u_{0}+w}{2}} \frac{\omega^{n}}{n !}\right)}{\sin \left(\arccos \left(4 \int_{M} e^{\frac{u_{0}+w}{2}} \frac{\omega^{n}}{n !}\right)\right)} .
$$

It remains only to check that $v$ is in the domain of definition of the exponential map, i.e. $\operatorname{arccot}\left(\frac{-\min v}{2|v|_{u_{0}}}\right)-|v|_{u_{0}}>0$. Notice that

$$
\left.-\min v=\left(4 \int_{M} e^{\frac{u_{0}+w}{2}} \frac{\omega^{n}}{n !}\right)-\min e^{\frac{w-u_{0}}{2}}\right) \frac{2 \arccos \left(4 \int_{M} e^{\frac{u_{0}+w}{2}} \frac{\omega^{n}}{n !}\right)}{\sin \left(\arccos \left(4 \int_{M} e^{\frac{u_{0}+w}{2}} \frac{\omega^{n}}{n !}\right)\right)}
$$

thus

$$
\operatorname{arccot}\left(\frac{-\min v}{2|v|_{u_{0}}}\right)=\operatorname{arccot}\left(\frac{4 \int_{M} e^{\frac{u_{0}+w}{2}} \frac{\omega^{n}}{n !}-\min e^{\frac{w-u_{0}}{2}}}{\sin \left(\arccos \left(4 \int_{M} e^{\frac{u_{0}+w}{2}} \frac{\omega^{n}}{n !}\right)\right)}\right)
$$

Also

$$
|v|_{u}=\operatorname{arccot}\left(\frac{4 \int_{M} e^{\frac{u_{0}+w}{2}} \frac{\omega^{n}}{n !}}{\sin \left(\arccos \left(4 \int_{M} e^{\frac{u_{0}+w}{2}} \frac{\omega^{n}}{n !}\right)\right)}\right)
$$

since the function arccot is decreasing and $\frac{-\min e^{\frac{w-u_{0}}{2}}}{\sin \left(\arccos \left(4 \int_{M} e^{\frac{u_{0}+w}{2}} \frac{\omega^{n}}{n !}\right)\right)}<0$, conclude

$$
\operatorname{arccot}\left(\frac{4 \int_{M} e^{\frac{u_{0}+w}{2}} \frac{\omega^{n}}{n !}-\min e^{\frac{w-u_{0}}{2}}}{\sin \left(\arccos \left(4 \int_{M} e^{\frac{u_{0}+w}{2}} \frac{\omega^{n}}{n !}\right)\right)}\right)-\operatorname{arccot}\left(\frac{4 \int_{M} e^{\frac{u_{0}+w}{2}} \frac{\omega^{n}}{n !}}{\sin \left(\arccos \left(4 \int_{M} e^{\frac{u_{0}+w}{2}} \frac{\omega^{n}}{n !}\right)\right)}\right)>0
$$

as wanted.

Lemma 5.5 Let $a \in \mathbb{R}$ and $\gamma\left(t, u_{0}, v_{0}\right)$ be as in Definition 5.3; then as long as both sides make sense we have

$$
\gamma\left(t, u_{0}, a v_{0}\right)=\gamma\left(a t, u_{0}, v_{0}\right)
$$


Proof. If $a$ or $t$ are zero, then the formula reads $u_{0}=u_{0}$; so consider the case when both $t$ and $a$ are non zero. The lefthand side reads

$$
\begin{gathered}
\gamma\left(t, u_{0}, a v_{0}\right)=u_{0}+2 \log \left(\cos \left(\left|a v_{0}\right|_{u_{0}} t\right)+\frac{a v_{0}}{2\left|a v_{0}\right|_{u_{0}}} \sin \left(\left|a v_{0}\right|_{u_{0}} t\right)\right)= \\
=u_{0}+2 \log \left(\cos \left(|a| \cdot\left|v_{0}\right|_{u_{0}} t\right)+\frac{\operatorname{sgn}(a) v_{0}}{2\left|v_{0}\right|_{u_{0}}} \sin \left(|a| \cdot\left|v_{0}\right|_{u_{0}} t\right)\right)= \\
=u_{0}+2 \log \left(\cos \left(\left|v_{0}\right|_{u_{0}} a t\right)+\frac{\operatorname{sgn}(a) v_{0}}{2\left|v_{0}\right|_{u_{0}}} \operatorname{sgn}(a) \sin \left(\left|v_{0}\right|_{u_{0}} a t\right)\right)= \\
=u_{0}+2 \log \left(\cos \left(\left|v_{0}\right|_{u_{0}} a t\right)+\frac{v_{0}}{2\left|v_{0}\right|_{u_{0}}} \sin \left(\left|v_{0}\right|_{u_{0}} a t\right)\right)=\gamma\left(a t, u_{0}, v_{0}\right),
\end{gathered}
$$

as claimed, where a property of the functions sinus and cosine is used.

The following result shows an important property shared by our and the standard exponential maps.

Lemma 5.6 Fix a point $u \in \mathcal{C}$. The differential of the exponential map at the origin is the identity operator.

Proof. Let $v \in T_{u} \mathcal{C}$ be any point in the domain of the exponential map. By definition the differential is

$$
(d \exp )_{0} v:=\left.\frac{d}{d t}\right|_{t=0} \exp (t v)=\frac{d}{d t}_{\mid t=0} \gamma(1, u, t v)=\frac{d}{d t}_{\mid t=0} \gamma(t, u, v)=v
$$

where we used the Lemma 5.5.

Comparison with the Mabuchi-Semmes-Donaldson metric 5.7 In that case, as proved by S. Semmes [20] and S. Donaldson [15], the geodesic equation is equivalent to a homogeneous complex Monge-Ampère equation on $M \times[0,1] \times$ $S^{1}$. About the Cauchy problem, S. Donaldson in [15] constructed examples of short time existence for the Cauchy problem which cannot be extended and also examples where the Cauchy problem for geodesics does not admit solution even for short time. As a consequence, a definition of exponential map is not at hand as in our case.

\section{The Dirichlet problem for the geodesic equation}

In this section we prove that the Dirichlet problem has explicit unique smooth solution. We think that this was known to Eugenio Calabi. Moreover, the space 
$\mathcal{C}$ endowed with the Calabi's metric is a genuine metric space, with a smooth distance function. The geodesic lines realize the minimum of the length among smooth paths joining two fixed endpoints. To conclude, we compute that the diameter of the space is $\frac{\pi}{2} R$.

Theorem 6.1 For any two points $u_{0} \neq u_{1} \in \mathcal{C}$ the geodesic line explicitly given by

$$
e^{\frac{u(t)}{2}}=e^{\frac{u_{0}}{2}}\left(\cos (t)+e^{\frac{u_{1}-u_{0}}{2}} \frac{\sin (t)}{\sin \left(t_{0}\right)}-\cos \left(t_{0}\right) \frac{\sin (t)}{\sin \left(t_{0}\right)}\right),
$$

is the unique (modulo parametrization) solution of the Dirichlet problem

$$
\left\{\begin{array}{c}
D_{t} u^{\prime}=0 \\
u(0)=u_{0} \\
u\left(t_{o}\right)=u_{1},
\end{array}\right.
$$

where $t_{0} \in\left(0, \frac{\pi}{2}\right)$ is the unique solution of $\int_{M} e^{\frac{u_{0}+u_{1}}{2}} \frac{\omega^{n}}{n !}=\frac{1}{4} \cos \left(t_{0}\right)$.

Proof. The first claim is that the smooth curve in the statement is indeed a geodesic. Its velocity, at $t=0$, is

$$
v_{0}=\frac{2}{\sin \left(t_{0}\right)}\left(e^{\frac{u_{1}-u_{0}}{2}}-\cos \left(t_{0}\right)\right)
$$

moreover check that $v_{0}$ is an element of the tangent space to $\mathcal{C}$ at $u_{0}$;

$$
\begin{gathered}
\int_{M} v_{0} e^{u_{0}} \frac{\omega^{n}}{n !}=\int_{M} \frac{2}{\sin \left(t_{0}\right)}\left(e^{\frac{u_{1}-u_{0}}{2}}-\cos \left(t_{0}\right)\right) e^{u_{0}} \frac{\omega^{n}}{n !}= \\
=\frac{2}{\sin \left(t_{0}\right)} \int_{M}\left(e^{\frac{u_{1}+u_{0}}{2}}-\cos \left(t_{0}\right) e^{u_{0}}\right) \frac{\omega^{n}}{n !}=\frac{2}{\sin \left(t_{0}\right)}\left(\int_{M}\left(e^{\frac{u_{1}+u_{0}}{2}}\right) \frac{\omega^{n}}{n !}-\frac{1}{4} \cos \left(t_{0}\right)\right)=0 .
\end{gathered}
$$

By the uniqueness of solutions for the Cauchy problem the claim follows. About $t_{0}$, employ the Hölder inequality to get

$$
0<\int_{M} e^{\frac{u_{0}+u_{1}}{2}} \frac{\omega^{n}}{n !} \leq\left(\int_{M}\left(e^{\frac{u_{0}}{2}}\right)^{2} \frac{\omega^{n}}{n !}\right)^{\frac{1}{2}}\left(\int_{M}\left(e^{\frac{u_{1}}{2}}\right)^{2} \frac{\omega^{n}}{n !}\right)^{\frac{1}{2}}=\frac{1}{2} \cdot \frac{1}{2}=\frac{1}{4}
$$

and the last inequality is an equality if and only if there exists a $\lambda \in \mathbb{R}$ for which $e^{u_{1}}=\lambda e^{u_{0}}$; but the constraint $\frac{1}{4}=\int_{M} e^{u_{1}} \frac{\omega^{n}}{n !}=\int_{M} e^{u_{0}} \frac{\omega^{n}}{n !}$ forces $\lambda$ to be 1 , that is $u_{0}=u_{1}$. When $t \in\left(0, \frac{\pi}{2}\right)$, the function $\frac{1}{4} \cos (t)$ is decreasing and surjective onto $\left(0, \frac{1}{4}\right)$. Thus there exists a unique $t_{0} \in\left(0, \frac{\pi}{2}\right)$ such that

$$
\frac{1}{4} \cos \left(t_{0}\right)=\int_{M} e^{\frac{u_{0}+u_{1}}{2}} \frac{\omega^{n}}{n !}
$$

Clearly the geodesic is smooth; about the uniqueness, suppose there are two geodesics connecting $u_{0}$ and $u_{1}$. Since we are interested in uniqueness modulo 
parametrization, normalize both geodesics into unitary ones. If $v_{0}, w_{0} \in T_{u_{0}} \mathcal{C}$ are the velocity of these two geodesics in $u_{0}$ and $s, t \in\left(-\frac{\pi}{2}, \frac{\pi}{2}\right)$ are values for which the point $u_{1}$ is reached respectively by the first and by the second geodesic, i.e.

$$
u_{1}=u_{0}+2 \log \left(\cos (s)+\frac{v_{0}}{2} \sin (s)\right)
$$

and

$$
u_{1}=u_{0}+2 \log \left(\cos (t)+\frac{w_{0}}{2} \sin (t)\right),
$$

then consider the difference

$$
0=2 \log \left(\frac{\cos (s)+\frac{v_{0}}{2} \sin (s)}{\cos (t)+\frac{w_{0}}{2} \sin (t)}\right)
$$

or equivalently

$$
\cos (s)+\frac{v_{0}}{2} \sin (s)=\cos (t)+\frac{w_{0}}{2} \sin (t)
$$

Multiply by $e^{u_{0}}$ to get $\frac{1}{4} \cos (t)=\frac{1}{4} \cos (s)$ which implies $t= \pm s$ and $\frac{v_{0}}{2}= \pm \frac{w_{0}}{2}$; so, if $w_{0}=v_{0}$ the two geodesics coincide, while if $w_{0}=-v_{0}$ one geodesic is parametrized backward respect to the other. The theorem is proved.

We introduce the following notation. If $\alpha$ is a smooth curve in $\mathcal{C}$ passing through $u_{0}, u_{1}$, we write the length of $\alpha$ between $u_{0}$ and $u_{1}$ as

$$
\mathcal{L}_{\alpha}\left(u_{0}, u_{1}\right)
$$

which does not dependent on the parametrization and is symmetric in $u_{0}$ and $u_{1}$.

Definition 6.2 Let $d$ be the map explicitly defined by

$$
\begin{aligned}
d: \mathcal{C} \times \mathcal{C} & \rightarrow\left[0, \frac{\pi}{2}\right) \\
\left(u_{0}, u_{1}\right) & \mapsto d\left(u_{0}, u_{1}\right):=\arccos \left(4 \int_{M} e^{\frac{u_{0}+u_{1}}{2}} \frac{\omega^{n}}{n !}\right)=\mathcal{L}_{u}\left(u_{0}, u_{1}\right),
\end{aligned}
$$

where $u$ is the geodesic line connecting $u_{0}$ and $u_{1}$, and as remarked in the Theorem 6.1 the argument of arccos varies between 0 and 1; moreover, the corresponding determination of the function arccos will range between 0 and $\frac{\pi}{2}$.

The next lemma is useful to prove that the function $d$ is a distance and that the geodesic distance is a minimum among the length of all smooth paths; we follow an argument by Xiu Xiong Chen [9].

Lemma 6.3 If $C$ is a smooth curve $C=\phi(s):[0,1] \rightarrow \mathcal{C}$, then for every $s \in[0,1]$ the length of the geodesic $t \mapsto u(s, t)$ from the base point $0 \in \mathcal{C}$ to $\phi(s)$ is not greater than the length of the geodesic $u(0, t)$ from the base point 0 to $\phi(0)$ plus the length of $C$ between $\phi(0)$ and $\phi(s)$; more precisely, there holds

$$
\mathcal{L}_{u(s, t)}(0, \phi(s)) \leq \mathcal{L}_{u(0, t)}(0, \phi(0))+\mathcal{L}_{\phi}(\phi(0), \phi(s)) .
$$


Proof. By definition the length of $C$ between $\phi(0)$ and $\phi(s)$ is

$$
\mathcal{L}_{\phi}(\phi(0), \phi(s))=\int_{0}^{s} \sqrt{<\phi_{s}(\tau), \phi_{s}(\tau)>_{\phi(\tau)}} d \tau
$$

where $\phi_{s}$ stands for the derivative respect to the parameter $s$. Suppose, without loss of generality, that $u(s, t):[0,1] \times[0,1] \rightarrow \mathcal{C}$ with $u(s, 0)=0, u(s, 1)=\phi(s)$, for every $s \in[0,1]$. By definition the length of $u(s, t)$ between 0 and $\phi(s)$ is

$$
\mathcal{L}_{u(s, t)}(0, \phi(s))=\int_{0}^{1} \sqrt{<u_{\tau}(s, \tau), u_{\tau}(s, \tau)>_{u(s, \tau)}} d \tau .
$$

Notice that by construction there holds

$$
u_{s}(s, 1)=\phi_{s}(s), \quad u_{s}(s, 0)=\phi_{s}(0) \forall s \in[0,1] .
$$

Define $F(s):=\mathcal{L}_{\phi}(\phi(0), \phi(s))+\mathcal{L}_{u(s, t)}(0, \phi(s))$, so that the claim is

$$
F(s)=\mathcal{L}_{\phi}(\phi(0), \phi(s))+\mathcal{L}_{u(s, t)}(0, \phi(s)) \geq \mathcal{L}_{u(s, t)}(0, \phi(0))=F(0) .
$$

To get the above claim, it is enough to show $\frac{\partial F}{\partial s} \geq 0$. Compute $\frac{\partial \mathcal{L}_{\phi}(\phi(0), \phi(s))}{\partial s}$;

$$
\begin{gathered}
\frac{\partial \mathcal{L}_{\phi}(\phi(0), \phi(s))}{\partial s}=\sqrt{<\phi_{s}(s), \phi_{s}(s)>_{\phi(s)}} \geq \\
\geq-<\phi_{s}(s), u_{t}(s, 1)>_{\phi(s)} \cdot\left(\sqrt{<u_{t}(s, 1), u_{t}(s, 1)>_{\phi(s)}}\right)^{-1},
\end{gathered}
$$

where the Cauchy-Schwartz inequality is used. Compute

$$
\begin{gathered}
\frac{\partial \mathcal{L}_{u(s, t)}(0, \phi(s))}{\partial s}=\int_{0}^{1} \frac{1}{2} \frac{1}{\sqrt{<u_{t}, u_{t}>_{u(s, \tau)}}} \cdot 2<D_{s} u_{t}, u_{t}>_{u(s, \tau)} d \tau= \\
=\int_{0}^{1} \frac{1}{\sqrt{<u_{t}, u_{t}>_{u(s, \tau)}}}<D_{t} u_{s}, u_{t}>_{u(s, \tau)} d \tau= \\
=\frac{1}{\sqrt{<u_{t}, u_{t}>_{u(s, \tau)}}} \int_{0}^{1}\left[\frac{\partial}{\partial t}<u_{s}, u_{t}>_{u}-<u_{s}, D_{t} u_{t}>_{u}\right] d \tau= \\
=\frac{1}{\sqrt{<u_{t}, u_{t}>_{u(s, \tau)}}}\left[<u_{s}(s, 1), u_{t}(s, 1)>_{\phi(s)}-<0, u_{t}(s, 0)>_{0}\right] .
\end{gathered}
$$

Thus

$$
\begin{gathered}
\frac{\partial F}{\partial s}=\frac{\partial \mathcal{L}_{\phi}(\phi(0), \phi(s))}{\partial s}+\frac{\partial \mathcal{L}_{u(s, t)}(0, \phi(s))}{\partial s} \geq \\
\geq\left(\sqrt{<u_{t}(s, 1), u_{t}(s, 1)>_{\phi(s)}}\right)^{-1}\left[-<\phi_{s}(s)+u_{s}(s, 1), u_{t}(s, 1)>_{\phi(s)}\right]=0,
\end{gathered}
$$

as claimed, where it is used that $\phi_{s}(s)=u_{s}(s, 1)$ for all the values of $s$. 
Theorem 6.4 The map $d$ is a smooth distance function and geodesics realizes the absolute minimum of the length over all $C^{1}$ paths.

Proof. The fact that $d$ is smooth and symmetric in $u_{0}$ and $u_{1}$ follows from its explicit expression

$$
d\left(u_{0}, u_{1}\right)=\arccos \left(4 \int_{M} e^{\frac{u_{0}+u_{1}}{2}} \frac{\omega^{n}}{n !}\right) .
$$

Suppose that $d\left(u_{0}, u_{1}\right)=0$; then by definition of $d$ the argument of arccos must be 1 . On its turn, as remarked in 6.1 , the equality $\int_{M} e^{\frac{u_{0}+u_{1}}{2}} \frac{\omega^{n}}{n !}=\frac{1}{4}$ holds if and only if $u_{0}=u_{1}$. So $d\left(u_{0}, u_{1}\right)=0$ implies $u_{0}=u_{1}$. To prove the triangular inequality, fix any $u_{0}, u_{1}, u_{2} \in \mathcal{C}$ and apply the Lemma 6.3 to the case when $s=1$ and $C=\phi$ is a geodesic from $\phi(0)=u_{1}$ and $\phi(1)=u_{2}$. The Lemma gives the formula

$$
\mathcal{L}_{u(1, t)}\left(0, u_{2}\right) \leq \mathcal{L}_{u(0, t)}\left(0, u_{1}\right)+\mathcal{L}_{\phi}\left(u_{1}, u_{2}\right) .
$$

Since we have the freedom to choose the base point $0 \in \mathcal{C}$ arbitrarily, with the choice $u_{0}=0$ the triangular inequality follows. About the minimizing property, for any smooth curve $C=\phi(s):[0,1] \rightarrow \mathcal{C}$, we want to show that the length of $C$ between $\phi(0)$ and $\phi(1)$ is greater than the geodesic distance from $\phi(0)$ to $\phi(1)$. This follows from the lemma proved above, applied to the case when $s=1$ and the base point 0 is in $\phi(s)$; in fact,

$$
d(\phi(1), \phi(0)) \leq d(\phi(1), \phi(1))+\mathcal{L}_{\phi}(\phi(0), \phi(1))=\mathcal{L}_{\phi}(\phi(0), \phi(1)),
$$

as wanted.

We conclude this section discussing the diameter of the space $\mathcal{C}$ endowed with the Calabi's metric. So far we proved that any two points $u_{0}, u_{1} \in \mathcal{C}$ have distance in the range $\left[0, \frac{\pi}{2}\right)$. We claim that the supremum is precisely $\frac{\pi}{2}$.

Definition 6.5 The diameter of the space of Kähler metrics endowed with the Calabi's metric is

$$
\operatorname{Diam}(\mathcal{C}, \mathrm{Ca}):=\sup _{u_{0}, u_{1} \in \mathcal{C}} d\left(u_{0}, u_{1}\right)
$$

Theorem 6.6 There exists a sequence $\left\{u_{k}\right\} \subset \mathcal{C}$ such that, for any $u \in \mathcal{C}$, we have

$$
\lim _{k \rightarrow \infty} d\left(u, u_{k}\right)=\frac{\pi}{2}
$$

In particular, the space $\mathcal{C}$ endowed with the Calabi's metric has diameter

$$
\operatorname{Diam}(\mathcal{C}, \mathrm{Ca})=\frac{\pi}{2}
$$


Proof. Fix any point $p \in M$; for any $k \in \mathbb{N}$ fix two balls $B_{r_{k}} \subset B_{R_{k}}$ centered $p$ with radii respectively $r_{k}<R_{k}$ to be determined. With a standard argument based on the partitions of unity (see for example [21]), get a real smooth function $f_{k}$ on $M$ such that

$$
\left\{\begin{array}{cc}
f_{k}(q)=1 & \text { when } q \text { belongs to } B_{r_{k}} \\
0 \leq f_{k}(q) \leq 1 & \text { when } q \text { belongs to } B_{R_{k}} \backslash B_{r_{k}} \\
f_{k}(q)=0 & \text { when } q \text { belongs to } M \backslash B_{R_{k}} .
\end{array}\right.
$$

Define

$$
\alpha_{k}:=\int_{M} f_{k} \frac{\omega^{n}}{n !} .
$$

Of course $\left|B_{r}\right| \leq \alpha \leq\left|B_{R}\right|$, where $|A|$ is the measure of the open set $A \subset M$. Let $\left\{\epsilon_{k}\right\}$ be some positive sequence which tends to zero; the sequence $u_{k}$ is defined by

$$
e^{u_{k}}:=\frac{\frac{f_{k}}{4 \alpha_{k}}+\epsilon_{k}}{1+\epsilon_{k}} .
$$

For any $k, u_{k}$ is smooth and satisfies $\int_{M} e^{u_{k}} \frac{\omega^{n}}{n !}=\frac{1}{4}=\operatorname{Vol}(M)$. Fix the radii such that $\left|B_{r_{k}}\right|=\frac{1}{4^{k}},\left|B_{R_{k}}\right|=\frac{1}{3^{k}}$. Thus we have

$$
\begin{gathered}
0<\int_{M} e^{\frac{u_{k}}{2}} \frac{\omega^{n}}{n !} \leq \frac{1}{\sqrt{1+\epsilon_{k}}}\left[\left|M \backslash B_{R_{k}}\right| \sqrt{\epsilon_{k}}+\frac{\left|B_{R_{k}}\right|}{\sqrt{\alpha_{k}}} \sqrt{\frac{1}{4}+\epsilon_{k} \alpha_{k}}\right] \leq \\
\leq \frac{1}{\sqrt{1+\epsilon_{k}}}\left[\frac{1}{4} \sqrt{\epsilon_{k}}+\left(\frac{2}{3}\right)^{k} \sqrt{\frac{1}{4}+\epsilon_{k} \alpha_{k}}\right] \rightarrow 0,
\end{gathered}
$$

when $k \rightarrow \infty$. So if $u$ is any point of $\mathcal{C}$,

$$
\begin{gathered}
d\left(u, u_{k}\right)=\arccos \left(4 \int_{M} e^{\frac{u+u_{k}}{2}} \frac{\omega^{n}}{n !}\right) \geq \\
\geq \arccos \left(4 \max _{M}(u) \int_{M} e^{\frac{u_{k}}{2}} \frac{\omega^{n}}{n !}\right) \rightarrow \arccos (0)=\frac{\pi}{2},
\end{gathered}
$$

which proves the statement.

Remark 6.7 If the volume of $M$ and thus the sectional curvature $R$ is not normalized, in general we have

$$
\operatorname{Diam}(\mathcal{C}, \mathrm{Ca})=\frac{\pi}{2} R
$$

Definition 6.8 We call the boundary of $\mathcal{C}$ and we denote it by $\partial \mathcal{C}$, the space

$$
\partial \mathcal{C}:=\left\{u \in C^{\infty}(M, \mathbb{R} \cup\{-\infty\}) \mid \int_{M} e^{u} \frac{\omega^{n}}{n !}=\int_{M} \frac{\omega^{n}}{n !}, \exists x \in M: u(x)=-\infty\right\} .
$$


Theorem 6.9 For any fixed $u_{0} \in \mathcal{C}$ there exists a sequence $\left\{w_{k}\right\} \subset \partial \mathcal{C}$ such that

$$
d\left(u_{0}, w_{k}\right) \rightarrow 0 .
$$

In particular the distance of $u_{0} \in \mathcal{C}$ from the boundary $\partial \mathcal{C}$ is zero.

Proof. A unitary geodesic starting from $u_{0}$ with initial velocity $v_{k} \in T_{u_{0}} \mathcal{C}$ has explicit expression $e^{\frac{u(t)}{2}}=e^{\frac{u_{0}}{2}}\left(\cos (t)+\frac{v_{k}}{2} \sin (t)\right)$. The parameter $t$ corresponds to the distance between $u_{0}$ and $u(t)$; moreover, when $0 \leq t=\operatorname{arccot}\left(-\frac{\min \left(v_{k}\right)}{2}\right)$, then $u(t)$ belongs to the boundary $\partial \mathcal{C}$. With a technique very similar to that one employed in the proof of Theorem 6.6, is possible to construct a sequence of unitary vectors $\left\{v_{k}\right\}_{k} \subset T_{u_{0}} \mathcal{C}$ such that $\lim _{k \rightarrow \infty} \min v_{k}=-\infty$. We claim that with such a sequence we are able to define a sequence of points of the boundary of $\mathcal{C}$ such that the distance rom $u_{0}$ goes to zero. In fact, define, for all $k \in \mathbb{N}$,

$$
w_{k}:=u\left(t_{k}\right) \in \partial \mathcal{C}
$$

and compute $d\left(u_{0}, w_{k}\right)=d\left(u_{0}, u\left(t_{k}\right)\right)=t_{k}=\operatorname{arccot}\left(-\frac{\min v_{k}}{2}\right)$, so that

$$
\lim _{k \rightarrow \infty} d\left(u_{0}, w_{k}\right)=0
$$

as requested by the statement.

Comparison with the Mabuchi-Semmes-Donaldson metric 6.10 In that case the construction of the Chen's geodesics is completely different from ours. In fact Xiu Xiong Chen [9] had to deal with a degenerate fully non linear second order partial differential equation on the manifold $M \times S^{1} \times[0,1]$. He used the continuity method; thus he wrote a family of Monge-Ampère equations, depending on a parameter $\lambda$, such that at $\lambda=0$ the equation was the geodesic one whose solvability was required to be shown. The method consisted of proving that the set of $\lambda \in[0,1]$ whose associated problem admits a solution is not empty, open and closed. The first two points were not difficult to prove, since non degenerate Monge-Ampère on manifold with boundary had already been understood. The crucial point was about the closedness, i.e. the proof of a priori estimates for the solutions. The $C^{0}$ bound from above was given by the use of the maximum principle, while the bound from below was got by a trick that goes back to $\mathrm{E}$. Calabi. The $C^{0}$ estimate were enough to get interior estimates from above of the Laplacian; for this Chen employed a lemma by Yau. The estimates of the Laplacian from below were immediate. Thus Chen passed to consider the remaining case, i.e. the estimates form above of the Laplacian at the boundary of the manifold. About this Chen first proved that the mixed derivatives with a tangential component dominates the other ones; then, with a surprising barrier function, Chen showed that those derivatives are controlled by the gradient norm. The 
last step was to control the gradient of the norm from above. This step would give also the Laplacian estimates, for what we just said. The estimate of the gradient of a solution was got via a blowing-up analysis argument. Chen's theorem partially fulfills one conjecture in Donaldson's program [15], since Chen's geodesics are $C^{1,1}$ and it is not known yet if they are smooth. Moreover, it is still not known if Chen's geodesics lay completely inside the space, as in our case. Again by a Xiu Xiong Chen's theorem in [9], the space of Kähler metric endowed with that metric is a genuine metric space, and geodesics minimize the length among smooth paths. This completely fulfills another conjecture in Donaldson's program [15]. We got the same result in the Proposition 6.4, with the difference that in our case the distance function is smooth and in that case is $C^{1}$.

\section{$7 \quad$ The Jacobi equation; $\mathcal{C}$ as a portion of a sphere}

In this section we use the nice expression of geodesic lines and sectional curvature to define Jacobi fields and conjugate points in the same way of Riemannian geometry. We give a characterization and a link between conjugate points and the exponential maps; we prove that there are no conjugate points. Finally we show an isometric immersion of $\mathcal{C}$ in a portion of the sphere in $C^{\infty}(M, \mathbb{R})$.

Definition 7.1 Let $u=u(t):(-\epsilon, \epsilon) \rightarrow \mathcal{C}$ be a geodesic, and let $J=J(t)$ be a smooth section along $u$. We call $J$ a Jacobi field if it satisfies the Jacobi equation

$$
D_{t}^{2} J-R\left(u^{\prime}, J\right) u^{\prime}=0 .
$$

Remark 7.2 Fix a point $u_{0} \in \mathcal{C}$ in the space of Kähler metrics. Suppose to have a curve $v=v(s)$ in $\mathcal{V}$, and consider the two parameter family $(s, t) \mapsto \exp (t v(s))$, where we wrote $\exp$ instead $\exp _{u_{0}}$. Notice that, when $s$ is fixed, the curve $\alpha(t):=$ $\exp (t v(s))$ is a geodesic for any fixed $s$. In fact, by the Lemma 5.5 this curve can be expressed by

$$
t \mapsto \gamma\left(1, u_{0}, t v(s)\right)=\gamma\left(t, u_{0}, v(s)\right)
$$

which is precisely the form of a geodesic, by the definition of $\gamma$. We a smooth section $J$ along the curve $\alpha$ as

$$
J(t):=\mapsto \frac{\partial}{\partial s} \exp (t v(s)) .
$$

This is an example of a Jacobi field as we compute

$$
\begin{gathered}
D_{t}^{2} \frac{\partial}{\partial s} \exp (t v(s))=D_{t} D_{s} \frac{\partial}{\partial t} \exp (t v(s))= \\
=\left(D_{t} D_{s}-D_{s} D_{t}\right) \frac{\partial}{\partial t} \exp (t v(s))=R\left(\alpha^{\prime}, J\right) \alpha^{\prime},
\end{gathered}
$$

where we used in the last equality that $\alpha$ satisfies the geodesic equation. 
Lemma 7.3 For a Jacobi field $J$ along a geodesic u these two formulae hold $<u^{\prime}, D_{t} J>(t)=<u^{\prime}, D_{t} J>(0), \quad<u^{\prime}, J>(t)=<u^{\prime}, D_{t} J>(0) \cdot t+<u^{\prime}, J>(0)$.

Proof. First notice that

$$
\begin{gathered}
\frac{\partial}{\partial t}<u^{\prime}, D_{t} J>=<u^{\prime}, D_{t}^{2} J>=<u^{\prime}, R\left(u^{\prime}, J\right) u^{\prime}>= \\
=<u^{\prime},<u^{\prime}, u^{\prime}>J-<u^{\prime}, J>u^{\prime}>=0 .
\end{gathered}
$$

Thus $<u^{\prime}, D_{t} J>(t)=<u^{\prime}, D_{t} J>(0)$. About the second formula,

$$
\frac{\partial}{\partial t}<u^{\prime}, J>=<u^{\prime}, D_{t} J>(t)=<u^{\prime}, D_{t} J>(0),
$$

which gives, after an integration, the second claim.

Lemma 7.4 The Jacobi equation is equivalent to the second order differential equation

$J^{\prime \prime}+u^{\prime} J^{\prime}-2\left|v_{0}\right|_{u_{0}}^{2} J+4<v_{0}, D_{t} J(0)>_{u_{0}}+2\left(<v_{0}, D_{t} J(0)>_{u_{0}} t+<v_{0}, J(0)>{ }_{u_{0}}\right)=0$,

where $u(t)$ is a geodesic with initial data $\left(u_{0}, v_{0}\right)$ and $J$ is a Jacobi field along $u$.

Proof. In the next computation is assumed $R=1$;

$$
\begin{gathered}
D_{t}^{2} J=D_{t}\left(J^{\prime}+\frac{1}{2} u^{\prime} J+2<u^{\prime}, J>\right)=J^{\prime \prime}+\frac{1}{2} u^{\prime} J^{\prime}+2<u^{\prime}, J^{\prime}>+\frac{1}{2} u^{\prime \prime} J+ \\
+\frac{1}{2} u^{\prime} J^{\prime}+\frac{1}{4}\left(u^{\prime}\right)^{2} J+<u^{\prime}, u^{\prime} J>+2<u^{\prime}, J>+<u^{\prime}, J>u^{\prime}= \\
=J^{\prime \prime}+u^{\prime} J^{\prime}+2<u^{\prime}, J^{\prime}+\frac{1}{2} u^{\prime} J>+\frac{1}{2} J\left(u^{\prime \prime}+\frac{1}{2}\left(u^{\prime}\right)^{2}\right)+2<u^{\prime}, D_{t} J>+<u^{\prime}, J>u^{\prime}= \\
=J^{\prime \prime}+u^{\prime} J^{\prime}+4<u^{\prime}, D_{t} J>+\frac{1}{2} J\left(u^{\prime \prime}+\frac{1}{2}\left(u^{\prime}\right)^{2}\right)+<u^{\prime}, J>u^{\prime} \\
=J^{\prime \prime}+u^{\prime} J^{\prime}+4<u^{\prime}, D_{t} J>-<u^{\prime}, u^{\prime}>J+<u^{\prime}, J>u^{\prime} .
\end{gathered}
$$

Thus the Jacobi equation is equivalent to

$$
\begin{gathered}
D_{t}^{2} J-R\left(u^{\prime}, J\right) u^{\prime}= \\
=J^{\prime \prime}+u^{\prime} J^{\prime}+4<u^{\prime}, D_{t} J>-<u^{\prime}, u^{\prime}>J+<u^{\prime}, J>u^{\prime}-<u^{\prime}, u^{\prime}>J+<u^{\prime}, J>u^{\prime}= \\
=J^{\prime \prime}+u^{\prime} J^{\prime}+4<u^{\prime}, D_{t} J>-2<u^{\prime}, u^{\prime}>J+2<u^{\prime}, J>u^{\prime}= \\
=J^{\prime \prime}+u^{\prime} J^{\prime}+4<v_{0}, D_{t} J(0)>-2\left|v_{0}\right|_{u_{0}}^{2} J+2 u^{\prime}\left(<v_{0}, D_{t} J(0)>t+<v_{0}, J(0)>\right),
\end{gathered}
$$

where in the last equality the Lemma 7.3 is applied. The claim is proved. 
Lemma 7.5 Let $u$ be a geodesic in $\mathcal{C}$. If $J$ and $\tilde{J}$ are Jacobi fields such that $J(0)=\tilde{J}(0)$ and $D_{t} J(0)=D_{t} \tilde{J}(0)$ then $J=\tilde{J}$.

Proof. By the Lemma 7.4, the Jacobi equation is a second order differential equation for which the theorem on uniqueness of solution applies.

Proposition 7.6 Let $u=u(t)$ be a geodesic in $\mathcal{C}$ with initial velocity $v_{0} \in T_{u_{0}} \mathcal{C}$ and let $J$ be a Jacobi field along $u$ with $J(0)=0$ and $D_{t} J(0)=w$. Let $v(s)$ be a curve in $T_{u_{0}} \mathcal{C}$ with $v(0)=v_{0}$ and $v^{\prime}(0)=w$. Consider the Jacobi field $\tilde{J}(t)=\frac{\partial}{\partial s} s_{s=0} \exp (t v(s))$. Then $J=\tilde{J}$.

Proof. First notice that $w$ is an element of $T_{u_{0}} \mathcal{C}$ since it is $w=D_{t} J(0) \in T_{u_{0}} \mathcal{C}$. Also, $v^{\prime}(0)$ can be identified with an element of $T_{u_{0}} \mathcal{C}$; in fact, apply the operator $\frac{\partial}{\partial s \mid s=0}$ to the identity $\int_{M} v(s) e^{u_{0}} \frac{\omega^{n}}{n !}=0$ and get

$$
\int_{M} \frac{\partial v}{\partial s \mid s=0} e^{u_{0}} \frac{\omega^{n}}{n !}=0
$$

It is clear that $\tilde{J}(0)=0$; moreover notice that

$$
\begin{gathered}
D_{t} \frac{\partial}{\partial s}{ }_{s=0} \exp (t v(s))=D_{t}\left((d \exp )_{t v_{0}}(t w)\right)= \\
=D_{t}\left(t(d \exp )_{t v_{0}}(w)\right)=(d \exp )_{t v_{0}}(w)+t D_{t}\left((d \exp )_{t v_{0}}(w)\right) .
\end{gathered}
$$

In particular for $t=0$ the above formula reads

$$
D_{t} \tilde{J}(0)=(d \exp )_{0}(w)=w
$$

where the Lemma 5.6 is used. Conclude, by the Lemma 7.5, that $J=\tilde{J}$. $\square$

We want to show a link between the exponential map and the Jacobi fields that occurs as well in the finite dimensional theory. We start with the following notion

Definition 7.7 Let $u_{0}$ and $u_{1}$ be two points of $(\mathcal{C}, \mathrm{Ca})$, and $u=u(t)$ the geodesic that joins them so that $u(0)=u_{0}$ and $u\left(t_{0}\right)=u_{1}$. The two points $u_{0}, u_{1}$ are called conjugate when there is a Jacobi field $J$ along $u, J$ non identically zero, such that $J(0)=0$ and $J\left(t_{0}\right)=0$.

Proposition 7.8 Let $u=u(t)$ be a geodesic with $u(0)=u_{0}$ and $u^{\prime}(0) \neq 0$. The point $u_{1}=u\left(t_{0}\right)$ is conjugate to $u_{0}$ if and only if $v_{0}:=t_{0} u^{\prime}(0) \in T_{u_{0}} \mathcal{C}$ is a critical point of exp. 
Proof. With the use of the Proposition 7.6, write explicitly $J$ as

$$
J(t)=(d \exp )_{t u^{\prime}(0)}\left(t D_{t} J(0)\right)
$$

The field $J$ is not identically zero if and only if $D_{t} J(0) \neq 0$ as follows from the Lemma 7.5. Notice that $J(0)=0$; thus $u_{0}$ and $u_{1}$ are conjugate if and only if $J\left(t_{0}\right)=(d \exp )_{t_{0} u^{\prime}(0)}\left(t_{0} D_{t} J(0)\right)=0$, which means that $t_{0} u^{\prime}(0)$ is a critical point of exp.

We want to prove a statement about the existence of conjugate points in our case. First we need the following result.

Lemma 7.9 If $u_{0}, u_{1}$ are conjugate points and $J(0)=u_{0}, J(a)=u_{1}, J$ is not identically zero, necessarily we have

$$
<J, u^{\prime}>(t)=0, \quad<D_{t} J, u^{\prime}>(t)=0 .
$$

In particular the Jacobi equation simplifies into

$$
J^{\prime \prime}+u^{\prime} J^{\prime}-2\left|v_{0}\right|_{u_{0}}^{2} J=0 .
$$

Proof. Consider the second claim; from the Lemma 7.3

$$
<J, u^{\prime}>(t)=<D_{t} J(0), u^{\prime}(0)>t+<J(0), u^{\prime}(0)>.
$$

Use that $J(0)=0$ and evaluate the formula at $t=a$ to get $<J, u^{\prime}>(a)=<$ $D_{t} J(0), u^{\prime}(0)>a$. Since $J(a)=0$ the addendum $<D_{t} J(0), u^{\prime}(0)>$ vanishes and the formula simplifies into

$$
<J, u^{\prime}>(t)=<J(0), u^{\prime}(0)>,
$$

which is zero identically since $J(0)=0$. About the first claim, still from the Lemma $7.3,<u^{\prime}, D_{t} J>(t)=<u^{\prime}, D_{t} J>(0)$, which is already proved to be zero. Plug in the two formulae in the Lemma 7.4 to get the simplified equation.

Theorem 7.10 In the space $(\mathcal{C}, \mathrm{Ca})$ there are no conjugate points.

Proof. Suppose by contradiction that $u_{0}, u_{1} \in \mathcal{C}$ are conjugate points. So there exists a not identically zero Jacobi field $J$ along the geodesic $u$ which connects $u_{0}=u(0)$ and $u_{1}=u(a) . J$ necessarily satisfies the equation

$$
J^{\prime \prime}+u^{\prime} J^{\prime}-2\left|v_{0}\right|_{u_{0}}^{2} J=0
$$

Suppose, without loss of generality, that the geodesic which connects $u_{0}$ and $u_{1}$ is unitary. Thus the Jacobi equation simplifies into

$$
J^{\prime \prime}+u^{\prime} J^{\prime}-2 J=0
$$


Consider

$$
\left(e^{\frac{u}{2}} J\right)^{\prime \prime}=e^{\frac{u}{2}} J^{\prime \prime}+e^{\frac{u}{2}} J^{\prime} u^{\prime}+e^{\frac{u}{2}} J\left(\frac{u^{\prime \prime}}{2}+\frac{\left(u^{\prime}\right)^{2}}{4}\right)=e^{\frac{u}{2}} J^{\prime \prime}+e^{\frac{u}{2}} u^{\prime} J^{\prime}-e^{\frac{u}{2}} J .
$$

Thus the equation necessarily satisfied by $J$ is equivalent to the following one

$$
\left(e^{\frac{u}{2}} J\right)^{\prime \prime}-e^{\frac{u}{2}} J=0
$$

and the generic solution is

$$
e^{\frac{u}{2}} J=A \cos (t)+B \sin (t), \quad A, B \in \mathbb{R}
$$

The conditions $J(0)=J(a)=0$ and $J \neq 0$ entail

$$
A=0, \quad \sin (a)=0,
$$

that is $a=k \pi$, where $k \in \mathbb{Z}, k \neq 0$. But the geodesic $u$, exists at most in the interval $\left(-\frac{\pi}{2}, \frac{\pi}{2}\right)$, so a number $a=k \pi$, with $k \in \mathbb{Z}, k \neq 0$ cannot be contained in the interval of definition of $u$. This contradicts the fact that the Jacobi field $J$ exists for $t=a$. $\square$

Remark 7.11 The Theorem 7.10 is based on the fact that in the geometry arisen from the Calabi's metric, geodesic are not long enough to develop conjugate points. In Riemannian geometry a conjugate point is also linked to the point where a geodesic ceases to be a minimum of the length among smooth paths. Thus the above theorem is somehow consistent with the Proposition 6.4.

To conclude this section, we show an isometric immersion of $\mathcal{C}$ with the Calabi's metric into a portion of the sphere of $C^{\infty}(M, \mathbb{R})$ with a flat metric. Consider the space $C^{\infty}(M, \mathbb{R})$ endowed with the metric

$$
\prec \psi, \chi \succ_{\phi}:=\int_{M} \psi \chi \frac{\omega^{n}}{n !}, \quad \phi \in C^{\infty}(M, \mathbb{R}), \psi, \chi \in T_{\phi} C^{\infty}(M, \mathbb{R}),
$$

which is flat since there is not dependence on the point $\phi$ where the scalar product is computed. We call $\prec \cdot, \cdot \succ$ the Euclidean metric on $C^{\infty}(M, \mathbb{R})$.

Theorem 7.12 The map $A: \mathcal{C} \rightarrow C^{\infty}(M, \mathbb{R})$ defined as $A(u)=2 e^{\frac{u}{2}}$ is injective and has image $A(\mathcal{C})=\left\{f \in C^{\infty}(M, \mathbb{R}) \mid f>0, \int_{M} f^{2} \frac{\omega^{n}}{n !}=1\right\}$. Moreover, the pullback of the Euclidean metric on $C^{\infty}(M, \mathbb{R})$ via the map $A$ is the Calabi's metric. 
Proof. The differential of $A$ is

$$
(d A)_{u}(v)=\frac{d}{d t}{ }_{t=0} A\left(\exp _{u}(t v)\right)=2 e^{\frac{u}{2}} \frac{1}{2}\left(d \exp _{u}\right)_{0}(v)=e^{\frac{u}{2}} v .
$$

Thus the pullback is

$$
\left(A^{*} E u c l\right)[u](v, w)=\prec(d A)_{u}(v),(d A)_{u}(w) \succ_{A(u)}=\int_{M} e^{\frac{u}{2}} v e^{\frac{u}{2}} w \frac{\omega^{n}}{n !}=<v, w>_{u},
$$

as claimed.

Remark 7.13 The map $A$ is an isometric inclusion in analogy with the case of the isometric immersion of the part of the sphere $S^{2}$ lying in the first quadrant;

$$
\left\{x \in \mathbb{R}^{3} \mid x_{1}>0, x_{2}>0, x_{3}>0, \sum_{k=1}^{3} x_{k}^{2}=1\right\} \rightarrow\left(\mathbb{R}^{3}, \text { Eucl }\right) .
$$

Moreover, the diameter of this 2 dimensional pece of the sphere is $\frac{\pi}{2}$, consistently with the formula of the diameter. Everything scales correctly when the radius of the sphere is $R$ instead of 1 . Namely we have, when the radius of $\mathcal{C}$ is $R=\frac{1}{2 \sqrt{V o l}}$ with a generic value of the volume of $M$, that the map $A$ sends $\mathcal{C}$ in

$$
\left\{f \in C^{\infty}(M, \mathbb{R}) \mid f>0, \int_{M} f^{2}=R^{2}\right\}
$$

in this case the sectional curvature of $\mathcal{C}$ is $\frac{1}{R^{2}}$ and the diameter is $\frac{\pi}{2} R$.

\section{The Calabi's gradient metric}

Again from an idea by Calabi [8] comes the metric

$$
\ll \psi, \chi \gg_{\phi}:=\int_{M}(\nabla \psi, \nabla \chi)_{\phi} \frac{\omega_{\phi}^{n}}{n !}
$$

that we call the Calabi's gradient metric. It is a well defined metric on the space $\tilde{\mathcal{H}}$. We are going to show the existence of the Levi Civita covariant derivative for the Calabi's gradient metric. Then we are going to discuss the curvature induced by the Calabi's gradient metric and the geodesic equation in the case when $M$ is a closed Riemann surface. In the proof of the existence of the Levi Civita covariant derivative we will use the following basic result of Riemannian geometry due to W. Hodge, which we state without a proof. 
Lemma 8.1 Let $(X, g)$ be a closed Riemannian manifold of dimension $n$, and let $\Delta_{g}$ the Laplacian metric operator of the given Riemannian metric $g$. If a smooth real function $f \in C^{\infty}(X, \mathbb{R})$ satisfies

$$
\int_{M} f d \mu_{g}=0
$$

then there exists a smooth real function $h \in C^{\infty}(X, \mathbb{R})$ such that

$$
f=\Delta_{g} h .
$$

Proposition 8.2 The Levi Civita covariant derivative for the Calabi's gradient metric is given by

$$
2 \Delta_{\phi} D_{t} \psi=\frac{d}{d t}\left(\Delta_{\phi} \psi\right)+\Delta_{\phi} \psi^{\prime}+\left(\Delta_{\phi} \psi\right)\left(\Delta_{\phi} \phi^{\prime}\right),
$$

where $\phi$ is a smooth curve in $\tilde{\mathcal{H}}$ and $\psi$ is a smooth section along $\phi$.

Proof. We notice that the Calabi's gradient metric can be written, integrating by parts, as

$$
\ll \psi, \chi \gg_{\phi}=-\int_{M} \psi \Delta_{\phi} \chi \frac{\omega_{\phi}^{n}}{n !}=-\int_{M} \chi \Delta_{\phi} \psi \frac{\omega_{\phi}^{n}}{n !} .
$$

Thus, if $\phi=\phi(t)$ is a smooth path in the space of Kähler metrics and $\psi=\psi(t)$ is a smooth real section on $\phi$ we compute, to get the compatibility with the metric,

$$
\begin{gathered}
\frac{d}{d t}\left(-\int_{M} \psi \Delta_{\phi} \psi \frac{\omega_{\phi}^{n}}{n !}\right)=-\int_{M}\left(\psi^{\prime} \Delta_{\phi} \psi+\psi \frac{d}{d t}\left(\Delta_{\phi} \psi\right)+\psi\left(\Delta_{\phi} \psi\right)\left(\Delta_{\phi} \phi^{\prime}\right)\right) \frac{\omega_{\phi}^{n}}{n !}= \\
=-\int_{M} \psi\left(\Delta_{\phi} \psi^{\prime}+\frac{d}{d t}\left(\Delta_{\phi} \psi\right)+\left(\Delta_{\phi} \phi^{\prime}\right)\left(\Delta_{\phi} \psi\right)\right) \frac{\omega_{\phi}^{n}}{n !} .
\end{gathered}
$$

The computation here above suggests to define implicitly the Levi Civita covariant derivative as

$$
2 \Delta_{\phi} D_{t} \psi=\frac{d}{d t}\left(\Delta_{\phi} \psi\right)+\Delta_{\phi} \psi^{\prime}+\left(\Delta_{\phi} \psi\right)\left(\Delta_{\phi} \phi^{\prime}\right) .
$$

The expression on the righthand side has integral zero; in fact

$$
\int_{M} \frac{d}{d t}\left(\Delta_{\phi} \psi\right)+\Delta_{\phi} \psi^{\prime}+\left(\Delta_{\phi} \psi\right)\left(\Delta_{\phi} \phi^{\prime}\right) \frac{\omega_{\phi}^{n}}{n !}=\frac{d}{d t} \int_{M} \Delta_{\phi} \psi \frac{\omega_{\phi}^{n}}{n !}+\int_{M} \Delta_{\phi} \psi^{\prime} \frac{\omega_{\phi}^{n}}{n !}=0,
$$

where is just used integration by parts. Apply the Hodge result to get that there exists a function $A_{\phi}: T_{\phi} \tilde{\mathcal{H}} \rightarrow C^{\infty}(M, \mathbb{R})$ such that

$$
\Delta_{\phi} A=\frac{d}{d t}\left(\Delta_{\phi} \psi\right)+\Delta_{\phi} \psi^{\prime}+\left(\Delta_{\phi} \psi\right)\left(\Delta_{\phi} \phi^{\prime}\right)
$$


Now define $D_{t}: T_{\phi} \tilde{\mathcal{H}} \rightarrow T_{\phi} \tilde{\mathcal{H}}$ this way

$$
D_{t} \psi:=A_{\phi}(\psi)-L\left(0, A_{\phi}(\psi)\right) \text {. }
$$

Finally, it is straightforward to check the other properties of a Levi Civita covariant derivative.

When $M$ is a closed Riemann surface, the Levi Civita covariant derivative has the explicit formulation

$$
D_{t} \psi=\psi^{\prime}-\frac{1}{\mathrm{Vol}} \ll \psi, \phi^{\prime} \gg_{\phi}
$$

This makes possible an easy computation of the curvature tensor and of the geodesic curves

Proposition 8.3 When $M$ is a closed Riemann surface, the space of Kähler metrics endowed with the Calabi's gradient metric has zero curvature tensor and hence zero sectional curvature.

Proof. The second claim is a direct consequence of the first claim. To prove the first claim, we just remark that, if $\phi(s, t)$ is a smooth two parameter family in the space of Kähler metrics and $\psi$ is a section along it, then

$$
\begin{gathered}
D_{t} D_{s} \psi=D_{t}\left(\psi_{s}-\frac{1}{\mathrm{Vol}} \ll \psi, \phi_{s} \gg_{\phi}\right)=\psi_{t s}-\frac{1}{\mathrm{Vol}} \ll \psi_{s}, \phi_{t} \gg_{\phi}-\frac{d}{d t} \frac{1}{\mathrm{Vol}} \ll \psi, \phi_{s} \gg_{\phi}= \\
=\psi_{t s}-\frac{1}{\mathrm{Vol}}\left(\ll \psi_{s}, \phi_{t} \gg_{\phi}+\ll \psi_{t}, \phi_{s} \gg_{\phi}\right)-\frac{1}{\mathrm{Vol}} \ll \psi, \phi_{t s} \gg_{\phi}
\end{gathered}
$$

which is symmetric on $s$ and $t$, that is the curvature tensor is zero as claimed.

Theorem 8.4 When $M$ is a closed Riemann surface, the solution of the Cauchy problem

$$
\left\{\begin{array}{l}
D_{t} \phi^{\prime}=0 \\
\phi(0)=\phi_{0} \\
\phi^{\prime}(0)=\phi_{0}^{\prime}
\end{array}\right.
$$

is

$$
\phi(t)=\frac{1}{2 V o l} \ll \phi_{0}^{\prime}, \phi_{0}^{\prime} \gg_{\phi_{0}} t^{2}+\phi_{0}^{\prime} t+\phi_{0} .
$$

Proof. Clearly the curve

$$
\phi(t)=\frac{1}{2 V o l} \ll \phi_{0}^{\prime}, \phi_{0}^{\prime} \gg_{\phi_{0}} t^{2}+\phi_{0}^{\prime} t+\phi_{0}
$$


solves the differential problem; thus it remains only to check that the curve lies inside $\tilde{\mathcal{H}}$, that is $\int_{M} \phi^{\prime}(t) \omega_{\phi(t)}=0$. In fact

$$
\begin{gathered}
\int_{M} \phi^{\prime}(t) \omega_{\phi(t)}=\int_{M}\left(\frac{1}{V o l} \ll \phi_{0}^{\prime}, \phi_{0}^{\prime} \gg_{\phi_{0}} t+\phi_{0}^{\prime}\right)\left(1+t \Delta_{\phi_{0}} \phi_{0}^{\prime}\right) \omega_{\phi_{0}}= \\
=\ll \phi_{0}^{\prime}, \phi_{0}^{\prime} \gg_{\phi_{0}} t+\int_{M} \phi_{0}^{\prime} \omega_{\phi_{0}}+\frac{1}{V o l} \ll \phi_{0}^{\prime}, \phi_{0}^{\prime} \gg_{\phi_{0}} t^{2} \int_{M} \Delta_{\phi_{0}} \phi_{0}^{\prime} \omega_{\phi_{0}}+\int_{M} t \phi_{0}^{\prime} \Delta_{\phi_{0}} \phi_{0}^{\prime} \omega_{\phi_{0}}= \\
=t\left(\ll \phi_{0}^{\prime}, \phi_{0}^{\prime} \gg_{\phi_{0}}-\int_{M} t \phi_{0}^{\prime} \Delta_{\phi_{0}} \phi_{0}^{\prime} \omega_{\phi_{0}}\right)=0,
\end{gathered}
$$

where is used that $\int_{M} \phi_{0}^{\prime} \omega_{\phi_{0}}=0$ and that clearly $\int_{M} \Delta_{\phi_{0}} \phi_{0}^{\prime} \omega_{\phi_{0}}=0$. Notice that the addendum of second degree in $t$ does not depend on space variables, thus at the level of Kähler metrics the expression of the geodesic is affine in $t$ as expected when the curvature is zero.

\section{References}

[1] C. Arezzo and G. Tian, Infinite geodesic rays in the space of Kähler potentials, Ann. Sc. Norm. Super. Pisa Cl. Sci. (5) 2 (2003), no. 4, 617-630.

[2] A. Besse, Einstein manifolds, Classics in Mathematics. Berlin: Springer, 1987.

[3] J.P. Bourguignon, Ricci curvature and measures, Japan. J. Math. 4, 27-45 (2009).

[4] E. Calabi, The space of Kähler metrics, , Proc. Internat. Congr. Math. (Amsterdam, 1954), Vol 2, Noordhoff, Groningen, and North-Holland, Amsterdam, 1956, pp. 206-207.

[5] E. Calabi, Extremal Kähler metrics, In: "Seminar on Differential Geometry", Ann. Math. Stud. 102 (1982), 259-290.

[6] E. Calabi, Extremal Kähler metrics II, In: "Differential Geometry and Complex analysis", Lecture notes in Math., Springer, 1985, 96-114.

[7] E. Calabi and X.X. Chen, The space of Kähler metrics II, J. Differential Geom. 56 (2000), 189-234. 
[8] Private communication between X.X. Chen and E. Calabi.

[9] X.X. Chen, The space of Kähler metrics, J. Differential Geom. 61 (2002), 173-193.

[10] X.X. Chen, Space of Khler metrics, III. On the lower bound of the Calabi energy and geodesic distance., Invent. Math. 175 (2009), no. 3, 453-503.

[11] X.X. Chen, Space of Khler metrics, IV. On the lower bound of the K-energy. Preprint.

[12] X.X. Chen, W. He, The space of volume forms Preprint.

[13] X.X. Chen; S. Sun, Space of Kähler metrics (V). Kähler quantization. Preprint.

[14] X.X. Chen; G. Tian , Geometry of Kahler metrics and foliations by holomorphic discs , Publ. Math. Inst. Hautes Etudes Sci. No. 107 (2008), 1-107.

[15] S.K. Donaldson, Symmetric spaces, Kähler geometry and Hamiltonian dynamics, In: "Northern California Symplectic Geometry Seminar", 13-33, Amer. Math. Soc. Transl. (2) 196 Amer.Math. Soc., Providence, RI , 1999.

[16] S.K. Donaldson, Nahm's equation and free boundary problems, Preprint.

[17] T. Mabuchi, Some symplectic geometry on compact Kähler manifolds, Osaka J. Math. 24 (1999), 227-252.

[18] T. Mabuchi, K-energy maps integrating Futaki invariants, Tohoku Math. J. (2) 38 (1986), no. 4, 575-593.

[19] J. Milnor, Morse Theory, Princeton Uniersity Press, 1963.

[20] S. Semmes, Complex Monge-Ampère and symplectic manifolds, Amer. J. Math., 114 (1999), 495-550.

[21] F. W. Warner, Foundations of Differentiable Manifolds and Lie Groups, Springer-Verlag, 1983.

[22] S. T. Yau, On the Ricci Curvature of a Compact Kähler Manifold and the Complex Monge-Ampère Equation, Comm. P. And Appl. Math., 31(1978), 339-411. 


\section{Simone Calamai}

Dip. Matematica "U. Dini"

Università di Firenze

Viale Morgani 67/a

I-50134 Firenze - ITALY 